\title{
Retrato de uma região: impacto da indústria sucroalcooleira, indicadores de saúde e a percepção dos gestores
}

\section{Pictures from a region: impact of the sugarcane industry, health indicators and managers' perception}

\author{
Maria Silvia de Morais ${ }^{a}$ \\ ${ }^{\text {a} F a c u l d a d e ~ d e ~ M e d i c i n a ~ d e ~ S a ̃ o ~ J o s e ́ ~ d o ~ R i o ~ P r e t o . ~ D e p a r t a m e n t o ~}$ \\ de Epidemiologia e Saúde Coletiva. São José do Rio Preto, SP, \\ Brasil. \\ E-mail: msmoraes®famerp.br \\ José Carlos Lopes ${ }^{b}$ \\ ${ }^{b}$ Faculdade de Medicina de São José do Rio Preto. Departamento \\ de Epidemiologia e Saúde Coletiva. São José do Rio Preto, São \\ Paulo, Brasil. \\ E-mail: zekakauœuol.com.br

\section{Luciani Maria Vieira Rochac} \\ ${ }^{c}$ Centro Universitário Rio Preto. Faculdade de Arquitetura $e$ \\ Urbanismo. São José do Rio Preto, São Paulo, Brasil. \\ E-mail: arq.lucianirochaळgmail.com \\ Rafael Morais Chiaravalloti ${ }^{\mathrm{d}, e}$ \\ dUniversity College London. London, United Kingdom. \\ e'nstituto de Pesquisas Ecológicas. Nazaré Paulista, SP, Brasil. \\ E-mail: rafael.chiaravalloti.ı2ळucl.ac.uk

\section{Vânia Maria Sabadoto Brienze} \\ fFaculdade de Medicina de São José do Rio Preto. Departamento \\ de Ciências Neurológicas. São José do Rio Preto, São Paulo, Brasil. \\ E-mail: vania.brienzeœhospitaldebase.com.br
}

\section{Resumo}

Este artigo analisa indicadores de saúde dos municípios da região de São José do Rio Preto e a demanda da população segundo os gestores diante do processo de expansão da cana de açúcar. Dez municípios da região que possuem usinas de cana de açúcar e outros 10 municípios com o mesmo número de habitantes, mas sem usinas, foram selecionados. Os dados foram coletados por meio de entrevistas e em bancos de dados do Ministério da Saúde. Observou-se que, independentemente da presença de usinas sucroalcooleiras, houve melhora nos indicadores de saúde. Entretanto, para os gestores entrevistados, independentemente da presença ou ausência de usinas, a presença de migrantes para o corte da cana piorou os indicadores de saúde. Assim, constatou-se que a presença de usinas nos municípios não interferiu nos indicadores de saúde, mas os gestores consideram que a qualidade da saúde do município teve queda.

Palavras-chave: Saúde; Usinas Sucroalcooleiras; Sistema de Saúde em Cidades de Pequeno Porte; Indicadores de Saúde.

\section{Correspondência}




\section{Introdução}

This article analyzes health indicators in the municipalities of the São José do Rio Preto region (state of São Paulo, Brazil) and the demand of the population according to the managers regarding the sugar cane expansion process. Ten municipalities in the region that have sugar cane mills and 10 other municipalities with the same number of inhabitants, but without the presence of plants, were selected. Data were collected through interviews and Ministry of Health databases. It was observed that, regardless of the presence of sugar and alcohol plants, there was an improvement in health indicators. However, to the interviewed managers, despite there being plants or not, the presence of migrants to cut sugarcane worsened health indicators. Therefore, it was observed that the presence of plants in the municipalities did not interfere in health indicators, but managers perceived a drop in health quality in the municipality.

Keywords: Health; Sugarcane Industry; Health System in Small Cities; Health Indicators.
A cana-de-açúcar representa um importante produto do agronegócio brasileiro. A expansão da produção nacional começou com a criação do Programa Nacional Proálcool, em resposta à crise do petróleo em meados da década de 1970. O programa teve como principal objetivo criar um substituto para o petróleo que pudesse diminuir a dependência do país em relação a sua variação de preço (Pacca; Moreira, 2009).

As questões relacionadas ao aquecimento global foram a principal justificativa à efetivação do programa. Isso porque, ao longo do processo de produção, ao contrário do petróleo, não há retirada de material orgânico depositado e a posterior emissão de dióxido de carbono ( $\mathrm{CO}_{2}$ ) na atmosfera devido a sua queima. No entanto, diversos autores assinalaram que o produto brasileiro, ao longo do seu processo, teria impacto ambiental maior que a produção de petróleo. Esse efeito foi um dos principais fatores que não permitiu o Brasil adentrar em mercados internacionais (Lapola et al., 2010; Scarlemann e Laurance, 2008).

A falta de consenso sobre o impacto da cana-deaçúcar no meio ambiente provavelmente seja uma consequência de análises mais abrangentes sobre o assunto relacionado aos seus efeitos. Isso porque considerar apenas a diminuição na emissão de gases do efeito estufa, como os impactos da cana de açúcar, não configura uma realidade genérica (Chiaravalloti et al., 2014).

No estado de São Paulo a expansão de cana-deaçúcar se deu de maneira acelerada, principalmente na região de São José do Rio Preto - foco de nossa investigação. Originalmente, a região se consolidou como forte polo econômico devido à expansão da cultura cafeeira para o interior do estado de São Paulo. Hoje, constituída por ampla rede de infraestrutura viária, é uma das maiores áreas de plantação de cana-de-açúcar no estado, atingindo um terço de toda a área ocupada pelo agronegócio (Moraes; Priulli; Chiaravalloti, 2012).

Nessa região, a maior parte dos municípios apresenta população com menos de 10 mil habitantes, tendo grande número deles se tornado local de expressiva concentração de trabalhadores rurais em 
virtude da instalação da agroindústria canavieira (Bernardelli, 2003).

Em relação à saúde, essa área se destaca como amplo polo regional do estado de São Paulo. Entretanto, a região tem pouca tradição na gestão dos serviços, o que mantém os municípios historicamente dependentes técnica e politicamente da direção regional de saúde instaladas na cidadesede de São José do Rio Preto. Os municípios pequenos nos quais foram instaladas as usinas sucroalcooleiras, além de terem pouca capacidade de arrecadação, baixa competência técnica de gestão e inúmeras dificuldades para fixar profissionais de saúde, têm de arcar com financiamentos paralelos a fim de garantir atendimento para a população que aumentou nos últimos anos em razão da indústria canavieira (Chiaravalloti et al., 2014).

Com foco na gestão de saúde dos municípios da região de São José do Rio Preto, levantaram-se os indicadores de saúde e dos problemas enfrentados por seus gestores em vista da nova realidade da região, o agronegócio. Esse estudo faz parte de uma pesquisa que teve como objetivo entender o processo de expansão da cana-de-açúcar e seu impacto nos indicadores sociais da saúde e no meio ambiente entre esses municípios.

\section{Métodos}

Com o propósito de obter informações dos municípios da região de São José do Rio Preto sobre saúde, utilizaram-se dados secundários das Informações dos Municípios Paulistas (IMP) disponíveis na Fundação Sistema Estadual de Análise de Dados (Seade), dos anos 2010 e 2012. Para a análise, selecionamos 10 municípios que possuem usinas de cana-de-açúcar e outros 10 com o mesmo número de habitantes, mas que não possuíam usinas e não receberam trabalhadores migrantes para essa cultura.

Nas cidades selecionadas para o estudo, em 20 foram realizadas entrevistas com seis gestores de saúde, sendo três cidades em que há usinas de cana de açúcar e três que não possuem. As entrevistas tiveram o propósito de conhecer os equipamentos de saúde e a percepção sobre os problemas enfrentados na gestão do sistema local.
Esse projeto foi aprovado pelo Comitê de Ética em Pesquisa da Faculdade de Medicina de São José do Rio Preto (Famerp), sob Parecer nº 271.499, de 14 de maio de 2013.

\section{Resultados}

Segundo dados do Seade (2014), a região apresenta bons indicadores de saúde. A taxa de mortalidade infantil (por mil nascidos vivos) é por volta de 10,17\%, enquanto a do estado é de $13,44 \%$. Também obteve importante progresso na cobertura de serviços básicos de saúde, principalmente em relação à saúde reprodutiva. A proporção de mulheres que compareceram a sete ou mais consultas de pré-natal aumentou significativamente, bem como a diminuição de partos de mães adolescentes. Esse índice esteve abaixo dos percentuais para o Brasil (20,12\%), para a região Sudeste $(16,68 \%)$ e para o estado de São Paulo (14,84\%) no ano de 2012. 0 índice das taxas de cesarianas chama atenção na região de São José do Rio Preto, pois são quase sempre superiores a $70 \%$. A região apresentou taxa de $85,40 \%$.

Esses indicadores poucovariaram entre os municipios, não mostrando associações com a existência ou não de usinas de cana-de-açùcar. Entretanto, para os gestores, a presença dos migrantes para o corte de cana resultou em grande aumento dos problemas de saúde, principalmente os relacionados às gestantes e crianças, o que prejudica os indicadores, como mostra o seguinte depoimento: Aumentou demais a demanda por serviços, as crianças vêm de outros estados e com problemas de saúde, subnutridas. Os pais não têm informação nenhuma. São famílias diferentes. É outra cultura (E1).

Outro gestor entrevistado apresentou: Os migrantes trazem as crianças para serem atendidas quando a situação já está muito delicada, aí precisa de atendimento especializado, o gasto da secretaria é maior (E2). Ele afirma também que: As famílias da cidade são diferentes. O pessoal da saúde conhece todos. Eles são mais educados, nossos indicadores pioraram muito (E2).

Nas cidades em que não há usinas, os gestores também relataram aumento dos gastos em saúde relacionados às crianças: 
aumentaram muito os atendimentos depois que migrantes vieram morar nas cidades vizinhas, as mulheres deles acabam arrumando emprego aqui e trazem as crianças para os serviços de saúde em nossa cidade. Os problemas de saúde aumentaram e nossos indicadores pioraram. (E5)

Com relação à gravidez na adolescência, segundo os gestores, houve aumento significativo:

hoje, na cidade, tem muitas meninas grávidas, o pior que são de poucos recursos. Os pais não orientam e, como tem muito migrante, elas acabam engravidando. A secretária da assistência procura orientar, mas não adianta, não tem família estruturada. (Е3)

A prefeitura faz um serviço muito importante de orientação, nas escolas e nos postinhos, mas é muita gente de fora, parece que não estão muito preocupados se as filhas ficam grávidas. (E2)

Apesar de não ter usinas, vem muita gente morar aqui para trabalhar na região, não tem vínculo com a cidade. Só procuram os serviços de saúde na emergência. Aí nosso orçamento estoura. (E6)

Foi relatada também grande procura por remédios e consultas, e uma exigência, por parte da população migrante, por atendimento rápido e transporte em casos considerados como sem necessidade desse tipo de demanda. Afirmaram que há forte dependência desses serviços da cidade de São José do Rio Preto e que, apesar de o SUS preconizar uma integração de promoção, prevenção e assistência no processo de regionalização, na prática, isso não ocorre.

Segundo os gestores dos municípios, há escassez de equipamentos de cuidado à saúde para atender a população de modo geral e, em especial, os migrantes: Somos muito dependentes das cidades maiores (E2). Toda a população quer fazer exame e ir para uma cidade maior (E6).

Todos concordaram que o acesso universal a patamares equitativos de atenção à saúde não são garantidos. Salientaram que há pouco poder de decisão da esfera pública, alegando que o setor sucroalcooleiro exerce forte influência nas decisões locais da saúde. Nos municipios que não possuem usinas, os gestores relataram também a influência de setores da sociedade que interferem nas decisões dos programas de saúde. Para os gestores entrevistados, a esfera pública tem pouco poder de decisão, independentemente de possuir usinas ou não: $\varepsilon m$ cidades pequenas, vai muito do conhecimento que a pessoa tem para ter poder ( $\left.\mathrm{E}_{5}\right)$.

Com relação aos conselhos de saúde, os entrevistados alegam que ainda é inerte a participação da população, talvez pelo receio de represálias. Outro fator apontado pelos gestores foi a dificuldade dos conselheiros em controlar e acompanhar a aplicação dos recursos financeiros do município, em razão tanto da falta de informações como do desconhecimento dos programas municipais existentes.

Para os entrevistados, há grande dificuldade na oferta de serviços de saúde para a população de seus municípios: o repasse da União e do estado foram diminuindo enquanto a população e os problemas foram aumentando $\left(\mathrm{E}_{5}\right)$.

\section{Discussão}

Embora pontuais, os dados fornecem elementos capazes de auxiliar a elucidação do contexto das ações de saúde, permitindo identificar, de modo geral, em que medida a indústria sucroalcooleira interferiu nos indicadores de saúde dos municípios, bem como verificar a percepção dos gestores sobre esse processo.

Pela análise dos problemas enfrentados pelos gestores, pode-se constatar, por via de regra, em que proporção a teoria e a prática preconizadas vêm caminhando nesses municípios, isto é, como os princípios norteadores do Sistema Único de Saúde (SUS) estão sendo aplicados.

Os gestores relataram que a migração consequente da instalação da agroindústria canavieira é o maior problema enfrentado pela saúde dos municípios e alegam que não há entendimento dos procedimentos que lhes são preconizados pelo serviço de saúde. Segundo eles, essa incompreensão deve-se aos valores adquiridos em sua sociedade de origem, fundamentando em hábitos e práticas sociais "muito difíceis de serem modificadas”. Salientaram que a 
saúde das crianças e a gravidez na adolescência são os principais problemas.

Entretanto, avaliando os indicadores de saúde nos municípios, observou-se que esses não se alteraram na comparação proporcional entre os municípios que receberam migrantes e os que não receberam. Houve melhora dos indicadores em praticamente todas as cidades da região de São José do Rio Preto, independentemente da presença de usinas sucroalcooleiras.

Contudo, para os gestores, o processo migratório desencadeado pela indústria da cana causou muitos problemas de saúde nos municipios, principalmente os relacionados com a saúde reprodutiva das mulheres.

Migração é um importante fator de encontro entre atores sociais com diferentes histórias e valores. Muitas vezes, dentro desse contexto, certos conflitos são gerados e determinados preconceitos são expostos. No caso das mulheres, essa exposição é frequente, mesmo tendo seus valores fundamentados em seus contextos históricos, além de sofrerem uma carga de preconceito no que diz respeito ao seu local de origem, ficando mais vulneráveis nesse processo.

Diante desses dados, podemos afirmar que o processo de descentralização no SUS foi importante para aumentar a cobertura de saúde nos municípios, mas, muitas vezes, não conduziu a arranjos mais cooperativos na saúde. Após a promulgação da Constituição de 1988, que regulamentou o SUS, os municípios tornaram-se responsáveis por estruturar os serviços de atenção à saúde (Viana; Lima; Ferreira, 2010).

Os municípios da região que, em sua maioria, são pequenos, afora sua capacidade de arrecadação, têm baixa capacidade técnica de gestão e inúmeras dificuldades para fixar profissionais de saúde. Oferecem apenas atenção primária, institucionalizando profissionais de generalidades, quase sempre sem possibilidades de operar com uma clínica ampliada em sua competência de diagnosticar e curar.

Conforme Silva (2008), para minimizar as carências dos municípios - principalmente os de pequeno porte - é necessária a conformação de redes de integralidade do cuidado. 0 autor reconhece que isso não é possível às Redes de Atenção à Saúde (RAS) se não superarem obstáculos conjunturais e estruturais que dificultam maiores avanços nas politicas de saúde. Silva (2008) salienta que há necessidade de realizações micropolíticas articuladas com ações macropolíticas para que elas sejam efetivadas. Fleury e Ouverney (2007) afirmam que a rede poderá ser explorada se houver institucionalização para combinar as múltiplas capacidades dos atores ou organizações que dela fazem parte, principalmente para os municipios de pequeno porte.

De acordo com os gestores, há outra questão levantada: o pouco poder de decisão para implementar políticas públicas em seus municípios. Observou-se que os municípios passaram de prestadores de serviços em uma realidade que superpõe duas dimensões: uma estruturada em parâmetros sociais e outra baseada em práticas culturais e políticas locais. Essa superposição, frequentemente, leva a conflitos de interesse na distribuição de verbas, já que o centro de decisões fica muito próximo dos atores interessados (Machado; Fortes; Somarriba, 2004). Portanto, há necessidade de coordenação pelo poder central no processo de descentralização, vinculando procedimentos à aquisição de verbas.

Verificou-se também que os serviços de atenção à saúde operando no cotidiano são contraditórios entre os modelos preconizados e as práticas efetivamente exercidas. Assim, as ações de saúde nas quais protocolos, fluxos e rotinas se fundamentam podem se materializar em atos de cuidado por meio do trabalho, numa relação intersubjetiva, em que se inserem os sujeitos, e é nessa relação que aparecem as contradições (Ayres, 2009).

Com relação à saúde da mulher, apesar da melhora dos indicadores, há iniquidade no atendimento, principalmente às questões relacionadas à saúde reprodutiva, destacando-se as migrantes. Salientase a prática abusiva do parto cirúrgico (cesariana) cuja incidência vem aumentando na maioria das localidades brasileiras. A região de São José do Rio Preto apresenta as maiores taxas, representando maior risco tanto para a mãe como para o filho (Tavares, 2007). Para os gestores, as migrantes apresentam mais dificuldade de acesso aos serviços de saúde do que as moradoras do local.

A migração é um importante fator de encontro entre atores sociais com diferentes histórias e valores. 
Muitas vezes, no entanto, tal interação gera conflitos e expõe preconceitos. Mulheres que já sofrem uma carga de preconceitos no seu local de origem ficam mais vulneráveis diante desse processo.

Não se descarta que as diversidades de vivências das mulheres, no caso das migrantes, coexistem com diferentes formas de organização da sociedade. Fatores repercutem sobre o processo de viver/adoecer e de acesso aos serviços de saúde, conformando um mosaico de interesses, que carregam para os serviços de saúde toda essa diversidade.

Com o aumento dos processos de fluxo migratório entre os territórios, essas diversidades no atendimento à saúde reprodutiva das mulheres tende a transparecer. As migrantes trazem consigo uma bagagem de conhecimentos que foram fornecidos e interpretados por elas e, no processo de contato com um novo serviço de saúde (realidade apresentada), há reorganização dos significados de sua saúde e uma nova realidade é negociada. Isso torna o princípio de equidade, preconizado pelo SUS, fundamental para o atendimento da população e, em especial, das mulheres.

Os fatos demonstram que há mobilidade diferenciada na procura por serviços de saúde. Para algumas pessoas, as possibilidades de desfrutar dos espaços urbanos, de acessar simultaneamente os vários serviços com suas complexidades distribuidas nos espaços centrais são quase instantâneos, enquanto, para a grande maioria da população, incluindo os migrantes, o que predomina é a escassez e a demora.

No mesmo espaço e instante em que devemos buscar formas para que o SUS possa se consolidar como política de Estado duradoura e sustentável, não se podem perder de vista as diferentes racionalidades que perpassam os rumos efetivos desse sistema.

O processo de descentralização no SUS foi importante para aumentar a cobertura de saúde nos municípios, mas, muitas vezes, não conduziu a arranjos mais cooperativos na saúde. Após a promulgação da Constituição de 1988, que regulamentou o (SUS), os municípios tornaram-se responsáveis por estruturar os serviços de atenção à saúde (Viana; Lima; Ferreira, 2010).

A questão da saúde dos imigrantes, apesar de constituir um campo de direito e ser concebida como questão universal, pelo acesso ao SUS, não é garantia de acesso aos serviços. Observa-se uma iniquidade no atendimento a essa população devido à exclusão social vivenciada nesses locais. Essa é uma das principais causas da desigualdade no acesso aos serviços de saúde, tornando a população vulnerável ao agravo de problemas patológicos.

\section{Referências}

AYRES, J. R. C. M. Cuidado: trabalho e interação na práticas de saúde. Rio de Janeiro: Editora do Centro de Estudos e Pesquisa em Saúde Coletiva, 2009.

BERNARDELLI, M. L. F. H. Os papéis urbanos na região de Catanduva-SP: relações entre a produção de moradia e o trabalho volante. Scripta Nova: Revista Eletrónica de Geografía y Ciencias Sociales, Barcelona, v. 146, n. 81, 2003.

CHIARAVALLOTI, R. M. et al. Efeitos da expansão da cana de açúcar no sudeste do Mato Grosso do Sul e possíveis caminhos para uma agenda sustentável. Sustentabilidade em Debate, Brasília, DF, v. 5, n .1, p. 117-135, 2014.

FLEURY, S.; OUVERNEY, A. M. Gestão de redes: a estratégia de regionalização da política de saúde. Editora FVG, Rio de Janeiro, 2007.

LAPOLA, D. M. et al. Indirect land-use changes can overcome carbon savings from biofuels in Brazil. PNAS, Washington, DC, v. 107, n. 8, p. 3388-3393, 2010.

MACHADO, E. N. M.; FORTES, F. B. C. T.; SOMARRIBA, M. Efeitos da introdução do PAB sobre a distribuição de recursos e a prestação de serviços: caso de Minas Gerais. Ciência e Saúde Coletiva, Rio de Janeiro, v. 9, n. 1, p. 99-111, 2004.

MORAES, M. S.; PRIULLI, R. M. A.; CHIARAVALLOTI, R. M. A saúde e o jovem migrante. Cadernos de Saúde Coletiva, Rio de Janeiro, v. 20, n. 4, p. 440-447, 2012.

PACCA, S.; MOREIRA, J. R. Historical carbon budget of the Brazilian ethanol program. Energy Policy, Rio de Janeiro, v. 37, n. 11, p. 4863-4873, 2009.

SCARLEMANN, J. P. N.; LAURANCE, W. F. How green are biofuels? Science, Washington, DC, v. 319, p. 43-44, 2008. 
SEADE - FUNDAÇÃO SISTEMA ESTADUAL DE ANÁLISE DE DADOS. Informações dos municípios paulistas. São Paulo, 2014. Disponível em:

<https://goo.gl/QRHAqL>. Acesso em: 22 ago. 2016.

SILVA, S. F. Redes de atenção à saúde no SUS: o pacto pela saúde e redes regionalizadas de ações e serviços de saúde. Campinas: Instituto de Direito Sanitário Aplicado, 2008.
TAVARES, R. Construindo mapas conceituais. Ciências e Cognição, Rio de Janeiro, v. 12, p. 72-85, 2007.

VIANA, A. L.; LIMA, L. D.; FERREIRA, M. P. Condicionantes estruturais da regionalização na saúde: tipologia dos colegiados de gestão regional. Ciência e Saúde Coletiva, Rio de Janeiro, v. 15, n. 5, p. 2317-2326, 2010.

\section{Contribuição dos autores}

Todos os autores participaram da concepção do estudo, análise dos dados, redação e aprovaram a versão final do manuscrito.

Recebido: 07/11/2016

Reapresentado: 27/06/2018

Aprovado: 10/07/2018 